\title{
Repair-oriented classification of aortic insufficiency: Impact on surgical techniques and clinical outcomes
}

Munir Boodhwani, MD, MMSc, Laurent de Kerchove, MD, David Glineur, MD, Alain Poncelet, MD, Jean Rubay, MD, Parla Astarci, MD, Robert Verhelst, MD, Philippe Noirhomme, MD, and Gébrine El Khoury, MD

Objective: Valve repair for aortic insufficiency requires a tailored surgical approach determined by the leaflet and aortic disease. Over the past decade, we have developed a functional classification of AI, which guides repair strategy and can predict outcome. In this study, we analyze our experience with a systematic approach to aortic valve repair.

Methods: From 1996 to 2007, 264 patients underwent elective aortic valve repair for aortic insufficiency (mean age $-54 \pm 16$ years; $79 \%$ male). AV was tricuspid in 171 patients bicuspid in 90 and quadricuspid in 3 . One hundred fifty three patients had type I dysfunction (aortic dilatation), 134 had type II (cusp prolapse), and 40 had type III (restrictive). Thirty six percent (96/264) of the patients had more than one identified mechanism.

Results: In-hospital mortality was 1.1\% (3/264). Six patients experienced early repair failure; 3 underwent re-repair. Functional classification predicted the necessary repair techniques in $82-100 \%$ of patients, with adjunctive techniques being employed in up to $35 \%$ of patients. Mid-term follow up (median [interquartile range]: 47 [29-73] months) revealed a late mortality rate of $4.2 \%$ (11/261, 10 cardiac). Five year overall survival was $95 \pm 3 \%$. Ten patients underwent aortic valve reoperation (1 re-repair). Freedoms from recurrent $\mathrm{Al}(>2+)$ and from AV reoperation at 5 years was $88 \pm 3 \%$ and $92 \pm 4 \%$ respectively and patients with type I ( $82 \pm$ $9 \%$; $93 \pm 5 \%)$ or II $(95 \pm 5 \%$; $94 \pm 6 \%)$ had better outcomes compared to type III (76 $\pm 17 \% ; 84 \pm 13 \%)$.

Conclusion: Aortic valve repair is an acceptable therapeutic option for patients with aortic insufficiency. This functional classification allows a systematic approach to the repair of $\mathrm{Al}$ and can help to predict the surgical techniques required as well as the durability of repair. Restrictive cusp motion (type III), due to fibrosis or calcification, is an important predictor for recurrent $\mathrm{Al}$ following $\mathrm{AV}$ repair.

Earn CME credits at

http://cme.ctsnetjournals.org

In recent years, there has been increasing interest in valvesparing surgery to treat pathology of the aortic valve and root. Valve-sparing aortic root replacement, pioneered by David and Feindel ${ }^{1}$ and Yacoub and coworkers, ${ }^{2}$ has standardized the treatment of aortic root pathology and helped to lay the foundation for the application of repair techniques to the aortic valve. Patients with dilatation of the aortic root and ascending aorta, however, commonly have concomitant cusp pathology that requires treatment. ${ }^{3,4}$ Furthermore, aortic insufficiency (AI) may occur in the absence of aortic pathology as a result of primary cusp disease. ${ }^{5}$ Repair techniques for the aortic valve, particularly for cusp pathol-

From the Department of Cardiovascular and Thoracic Surgery, Cliniques Universitaires Saint-Luc, Brussels, Belgium.

Read at the Eighty-eighth Annual Meeting of The American Association for Thoracic Surgery, San Diego, Calif, May 10-14, 2008.

Received for publication May 5, 2008; revisions received July 30, 2008; accepted for publication Aug 31, 2008.

Address for reprints: Gébrine El Khoury, MD, Service de Chirurgie Cardiovasculaire et Thoracique, Cliniques Universitaires Saint-Luc UCL 90, Ave Hippocrate 10,

Brussels B-1200, Belgium (E-mail: elkhoury@chir.ucl.ac.be).

J Thorac Cardiovasc Surg 2009; 137:286-94

$0022-5223 / \$ 36.00$

Copyright (c) 2009 by The American Association for Thoracic Surgery

doi: $10.1016 /$ j.jtcvs.2008.08.054 ogy, remain heterogeneously and infrequently applied and have not been systemically characterized. A major limitation to the more generalized application of aortic valve repair techniques is the absence of a common framework for valve assessment to guide the approach to valve repair.

Important lessons in this regard may be learned from the development of mitral valve repair. The Carpentier classification ${ }^{6}$ of mitral valve insufficiency was responsible, in large part, for the development and generalized dissemination of repair techniques for the mitral valve, because it provided a common language for cardiologists, anesthesiologists, and surgeons to communicate about disease mechanisms and pathology. Key characteristics of that classification system were that it encompassed the entire spectrum of disease, it clarified and provided insight into the mechanism of insufficiency, it could be consistently applied with different assessment modalities (echocardiography and surgical assessment), it guided the repair techniques, and, finally, it provided a framework for the assessment of long-term outcome for differing mitral valve pathologic entities.

During the past decade, we have developed a similar classification of aortic valve insufficiency with these characteristics in mind. ${ }^{7}$ The purpose of this study was to describe our experience with aortic valve repair for $\mathrm{AI}$ according to this systematic approach and to evaluate this classification system specifically with respect to its ability to guide surgical repair and predict clinical outcome. 


\section{Abbreviations and Acronyms \\ $\mathrm{AI}=$ aortic insufficiency \\ STJ $=$ sinotubular junction \\ $\mathrm{VAJ}=$ ventriculoaortic junction}

\section{MATERIALS AND METHODS \\ Patient Population}

From December 1995 to March 2007, a total of 264 consecutive patients referred for nonemergency surgery with at least $2+$ aortic valve insufficiency underwent surgical procedures on the aortic valve, aortic root, and ascending aorta at a single center. These patients comprise the study cohort. Specifically, patients with type A aortic dissection were excluded. Data on surgical techniques and classification of AI were collected prospectively and analyzed retrospectively. The choice of surgical technique was entirely at the surgeon's discretion. Patients undergoing concomitant cardiac procedures were included.

\section{Classification of AI}

During a similar period, a classification system for AI according to mechanisms of disease and the repair techniques used was developed and applied to this patient population (Figure 1). ${ }^{7}$ This classification centers around the idea that the aortic valve, much like the mitral valve, consists of two major components, the aortic annulus and the valve leaflets. Unlike that of the mitral valve, however, the annulus of the aortic valve is not a single anatomic structure. The functional aortic annulus rather consists of two separate components, the ventriculoaortic junction (VAJ) and the sinotubular junction (STJ). As in the Carpentier classification of mitral valve disease, regurgitation associated with normal leaflet motion is designated as type I. Type I AI is largely due to lesions of the functional aortic annulus, with type 1a AI resulting from STJ enlargement and dilatation of the ascending aorta, type Ib resulting from dilatation of the sinuses of Valsalva and the STJ, type Ic resulting from dilatation of the VAJ, and type 1d resulting from cusp perforation without a primary functional aortic annular lesion. Type II AI is due to leaflet prolapse as a result of excessive cusp tissue or commissural disruption. Type III AI is due to leaflet restriction, which may be found in bicuspid, degenerative, or rheumatic valvular disease as a result of calcification, thickening, and fibrosis of the aortic valve leaflets.

Patients may have either single or multiple lesions contributing to their AI. For example, patients with isolated type Ib AI (from dilatation of the sinuses of Valsalva) are expected to have a central regurgitant jet. The presence of a sinus of Valsalva aneurysm with an eccentric AI jet therefore suggests concomitant leaflet prolapse (type II) or restriction (type III). Further assessment of leaflet anatomy can help to delineate more fully the different mechanisms contributing to AI.

\section{Surgical Techniques}

Intraoperative transesophageal echocardiography was routinely performed. All procedures were performed through a median sternotomy. The ascending aorta or proximal aortic arch was cannulated, along with either single, two-stage right atrial cannulation or bicaval venous cannulation, depending on the concomitant procedures being performed. After systemic heparinization, the aorta was crossclamped, and antegrade warm, blood cardioplegia was administered either through the aortic root or through direct coronary ostial cannulation after aortotomy. A transverse aortotomy was performed approximately $1 \mathrm{~cm}$ above the STJ, and three 4-0 polypropylene sutures were placed at the level of the commissures for exposure. The aortic valve was assessed for the mechanisms of AI, and the echocardiographic findings were corroborated.

Similar to those for the mitral valve, aortic valve repair techniques follow the broad principle of correcting the lesion identified according to the classification. In cases of leaflet repair, a functional aortic annuloplasty is added to stabilize the repair. Annuloplasty to the functional aortic annulus has to be performed both proximally (VAJ) and distally (STJ) and may be performed with or without an aortic prosthesis. At the VAJ, annuloplasty may be accomplished by a valve-sparing root replacement procedure (eg, aortic valve reimplantation) or by performing subcommissural annuloplasty. Of note, an aortic valve remodeling procedure requires subcommissural annuloplasty for proximal annular stabilization. Subcommissural annuloplasty is performed to stabilize the VAJ and reduce the width of the interleaflet triangle, as previously described elsewhere, ${ }^{5}$ with $2-0$ pledgeted valve sutures. Annuloplasty at the STJ may be performed with a Dacron polyester fabric aortic tube graft of the appropriate diameter or by placing plication sutures at the STJ. For type II lesions, leaflet prolapse can be corrected with a variety of previously described techniques, including triangular resection (with or without pericardial patch repair), leaflet plication, and free margin

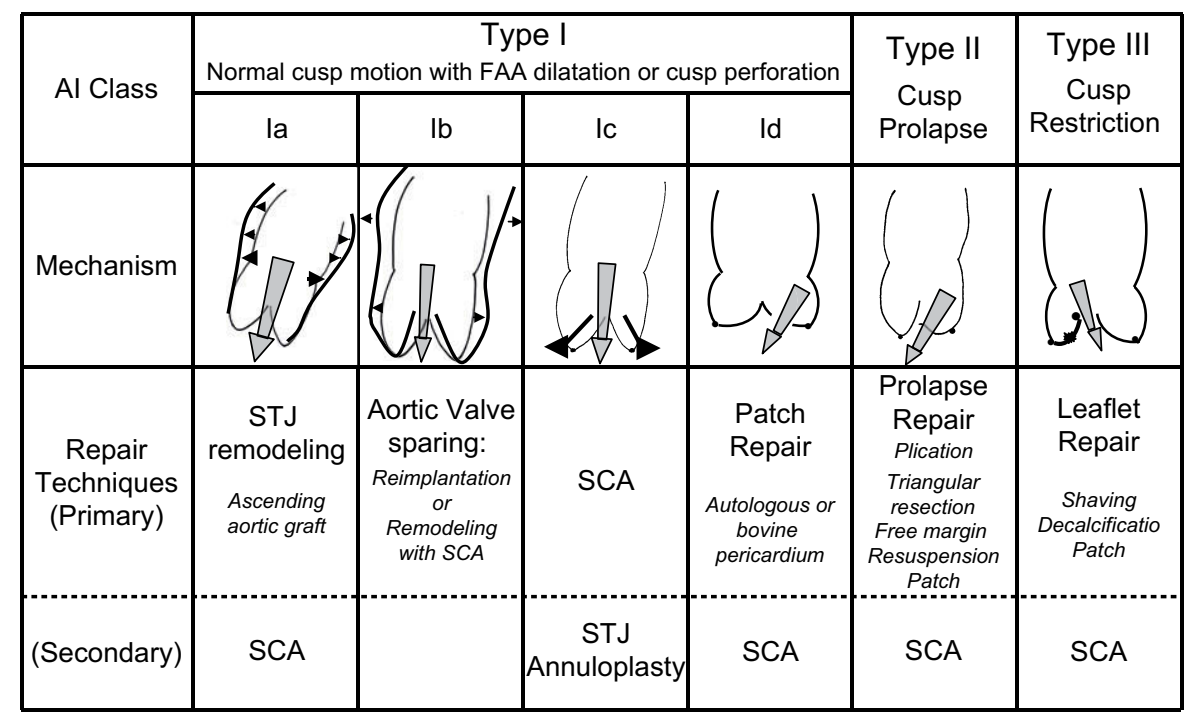

FIGURE 1. Repair-oriented functional classification of aortic insufficiency $(A I)$ with description of disease mechanisms and repair techniques used. FAA, Functional aortic annulus; STJ, sinotubular junction; SCA, subcommissural annuloplasty. 


\section{TABLE 1. Preoperative data}

\begin{tabular}{lc}
\hline Age (y, median and range) & $55(11-85)$ \\
Male sex (No.) & $209(79 \%)$ \\
New York Heart Association functional class (No.) & \\
I & $76(29 \%)$ \\
II & $130(49 \%)$ \\
III & $56(21 \%)$ \\
IV & $2(1 \%)$ \\
Previous cardiac surgery (No.) & $18(7 \%)$ \\
Left ventricular ejection fraction (No.) & \\
$>50 \%$ & $228(86 \%)$ \\
$30 \%-50 \%$ & $33(13 \%)$ \\
$<30 \%$ & $3(1 \%)$ \\
Left ventricular end-systolic diameter & $41 \pm 10$ \\
(mm, mean \pm SEM) & \\
Left ventricular end-diastolic diameter & $60 \pm 9$ \\
$\quad$ (mm, mean \pm SEM) & \\
Aortic insufficiency grade (No.) & \\
I & \\
II & $0(0 \%)$ \\
III & $67(25 \%)$ \\
IV & $153(58 \%)$ \\
Indication for surgery (No.) & $44(17 \%)$ \\
Aortic insufficiency & \\
Aortic insufficiency plus aortic dilatation & $113(43 \%)$ \\
Aortic diameter (mm, mean \pm SEM) & $151(57 \%)$ \\
Etiology (No.) & $53 \pm 9$ \\
Degenerative & \\
Bicuspid & $147(56 \%)$ \\
Marfan syndrome & $90(34 \%)$ \\
Endocarditis & $10(4 \%)$ \\
Other (rheumatic, aortitis, trauma, fibroelastoma) \\
\hline
\end{tabular}

resuspension. ${ }^{5}$ For type III or restrictive leaflet disease, shaving and decalcification of leaflets is required, with or without patching. The specific techniques recommended to address the mechanism for each class of AI are indicated in Figure 1. For each lesion type, the primary repair technique addresses the mechanism of the regurgitant lesion and the secondary technique typically involves stabilization of annulus through annuloplasty of the functional aortic annulus. In some cases, adjunctive repair techniques are used for intraoperatively discovered pathologic entities, not adequately diagnosed on preoperative echocardiography or for induced leaflet pathologic problems, such as induced leaflet prolapse after a valve-sparing root replacement procedure.

After the completion of repair, transesophageal echocardiography is critical for valve assessment. It was therefore performed in all patients specifically to assess the degree of AI, the orientation of the regurgitant jet (if present), and the coaptation length and coaptation level of the aortic valve cusps. Coaptation length of at least $5 \mathrm{~mm}$ at the midportion of the free margin and a coaptation level above the aortic valve annulus was a prerequisite for a successful repair, and the presence of an eccentric residual AI jet was an indication for reexploration of the aortic valve.

\section{Postoperative Care}

All patients were treated with aspirin after the operation and underwent transthoracic echocardiography before discharge.

\section{Follow-up}

Clinical follow-up was conducted either through outpatient visits or as telephone follow-up conducted by a research nurse. In addition to survival
TABLE 2. Operative and postoperative data

\begin{tabular}{lc}
\hline Cardiopulmonary bypass time (min, mean \pm SEM) & $114 \pm 5$ \\
Cardiac ischemic time (min) & $87 \pm 5$ \\
Aortic valve anatomy (No.) & \\
Tricuspid & $171(65 \%)$ \\
Bicuspid & $90(34 \%)$ \\
Quadricuspid & $3(1 \%)$ \\
Concomitant procedures (No.) & $85(32 \%)$ \\
Mitral valve repair & $41(16 \%)$ \\
Mitral valve replacement & $2(1 \%)$ \\
Tricuspid valve repair & $11(4 \%)$ \\
Coronary artery bypass grafting & $34(13 \%)$ \\
Aortic arch procedures & $7(3 \%)$ \\
Other (left ventricular aneurysm, atrial ablation, & $12(5 \%)$ \\
$\quad$ patent foramen ovale closure, myxoma) & \\
Postbypass transesophageal echocardiography (No.) & \\
No aortic insufficiency & $171(65 \%)$ \\
Grade I aortic insufficiency & $8(30 \%)$ \\
Grade II aortic insufficiency & $12(5 \%)$ \\
Repeat cardiopulmonary bypass for repair of residual & $14(5 \%)$ \\
aortic insufficiency (No.) & \\
Death (No.) & $3(1 \%)$ \\
Stroke (No.) & $1(0.4 \%)$ \\
Reoperation for bleeding or infection (No.) & $12(5 \%)$ \\
Need for pacemaker (No.) & $6(2 \%)$ \\
Hospital stay (d, median and range) & $6(5-7)$ \\
Discharge echocardiography (No.) & \\
No aortic insufficiency & $73(28 \%)$ \\
Grade I aortic insufficiency & $163(63 \%)$ \\
Grade II aortic insufficiency & $21(8 \%)$ \\
Grade III aortic insufficiency & $1(0.4 \%)$ \\
\hline &
\end{tabular}

status, information on valve-related complications including thromboembolism, hemorrhage, endocarditis, reoperation, and cardiovascular symptoms was obtained whenever possible. Transthoracic echocardiography was performed for all patients after discharge and at regular intervals during the course of follow-up. Echocardiograms not obtained at our institution were interpreted by the referring cardiologist.

\section{Statistical Analysis}

Data are presented as mean \pm SEM for continuous data or as median with interquartile range for nonparametric data. Failure time data for survival, reoperation, and recurrent AI are presented with Kaplan-Meier survival curves. Comparisons between groups for failure time data were performed with the log-rank test. Statistical analyses were performed with SAS version 9.1 software (SAS Institute, Inc, Cary, NC). Graphs were constructed with GraphPad Prism 4.0 (GraphPad Software, Inc, San Diego, Calif).

\section{RESULTS \\ Patient Population}

A total of 264 patients with significant AI $(\geq 2+)$ underwent aortic valve repair with or without aortic root, ascending aortic and concomitant cardiac procedures. Table 1 depicts the preoperative characteristics of study patients. The mean age was $54 \pm 16$ years, with about $80 \%$ of patients being male. More than $70 \%$ of patients had symptoms (New York Heart Association functional class $\geq$ II), and 


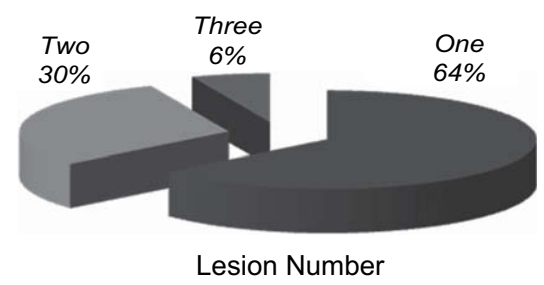

\begin{tabular}{|l|c|}
\hline \multicolumn{2}{|c|}{ Two Lesions $(\mathrm{n}=80)$} \\
\hline Type Ib and II & $38(48 \%)$ \\
\hline Type la and II & $14(18 \%)$ \\
\hline Type Ib and III & $10(12 \%)$ \\
\hline Type II and III & $10(12 \%)$ \\
\hline Type la and III & $3(4 \%)$ \\
\hline Type Ic and III & $2(3 \%)$ \\
\hline Type Id and II & $2(3 \%)$ \\
\hline Type Ib and Id & $1(1 \%)$ \\
\hline \multicolumn{2}{|c|}{ Three Lesions (n = 16) } \\
\hline Type Ib, II, and III & $11(69 \%)$ \\
\hline Type Ia, II, and III & $3(19 \%)$ \\
\hline Type Ib, Id, and II & $1(6 \%)$ \\
\hline Type Id, II, and III & $1(6 \%)$ \\
\hline
\end{tabular}

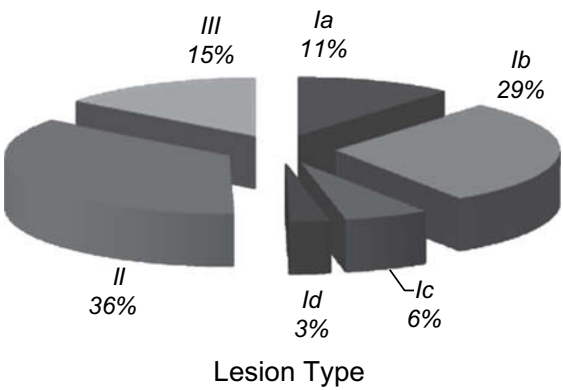

FIGURE 2. Description of aortic valve pathology according to number of lesions (top left), types of pathology observed (bottom left), and description and frequencies of multiple pathologies observed (right).

most had normal left ventricular ejection fractions. The most common etiology of AI was degenerative, followed by bicuspid aortic valve, Marfan syndrome, and endocarditis. For $43 \%$ of patients, the AI was the primary indication for surgery. For the remaining $53 \%$ of patients, who had both aortic dilatation and AI, the mean maximal aortic diameter was $53 \pm 9 \mathrm{~mm}$.

Intraoperative and postoperative data are presented in Table 2 . The cusp anatomy was tricuspid in $65 \%$, bicuspid in $34 \%$, and quadricuspid in $1 \%$. Postrepair intraoperative transesophageal echocardiography revealed AI grade II in 12 patients $(4.5 \%)$. This prevalence was increased to 21 patients $(8 \%)$ on discharge echocardiography. In-hospital mortality was $1.1 \%(n=3)$. Causes were multiorgan failure $(\mathrm{n}=1)$, respiratory failure $(\mathrm{n}=1)$, and congestive heart failure $(n=1)$. One patient had a stroke. Six patients underwent aortic valve reoperation during the index admission. In 3 of these cases, the valve was repaired again; the other 3 patients underwent aortic valve replacement. Of the patients who underwent another repair, 1 had an aorta-right ventricular fistula caused by tearing of the membranous septum by the subcommissural annuloplasty suture, and the other 2 had dehiscence of leaflet suture lines (1 direct suture of resected raphe and 1 valve patch). Of the patients who underwent aortic valve replacement, 1 had recurrent leaflet prolapse and 2 had significant recurrent AI caused by a lack of central coaptation. There were no deaths among patients undergoing aortic valve reoperation during the index admission.

\section{Classification of AI}

The classification of AI in the study cohort is presented in Figure 2. A total of 376 lesions were diagnosed in 264 patients. Approximately two thirds of patients were found to have solitary lesions. Two lesions were identified in $30 \%$ of patients and three in $6 \%$. Fifty percent of lesions were type I (normal leaflet motion with functional aortic annular dilatation or cusp perforation), $35 \%$ were type II (leaflet prolapse), and 15\% were type III (leaflet restriction). The most common sets of multiple lesions were prolapse of aortic valve leaflet in combination with type Ia (STJ dilatation, $\mathrm{n}=14$ ) or type $\mathrm{Ib}$ (aortic root aneurysm, $\mathrm{n}=38$ ) disease.

\section{Prediction of Surgical Techniques}

The ability of the classification system to predict the surgical technique used was evaluated by retrospectively comparing the predicted surgical technique with the actual technique used. For example, for isolated type Ia disease caused by STJ dilatation and ascending aortic aneurysm, the classification predicts STJ annuloplasty (typically with an aortic prosthesis), with subcommissural annuloplasty to stabilize the VAJ. We found that $100 \%$ of patients underwent STJ remodeling, and $82 \%$ underwent subcommissural annuloplasty. Similar predictability results for the other classifications are presented in Table 3. In addition to the predicted necessary corrective techniques, adjunctive techniques were also used in some cases to correct intraoperatively discovered pathology. For example, in type Ia disease, leaflet shaving $(\mathrm{n}=1)$ and quadricuspid leaflet repair $(n=1)$ were performed. The types and frequencies of adjunctive techniques used are also presented in Table 3. Overall, patients underwent surgical repair as predicted by the AI classification in $82 \%$ to $100 \%$ of cases, and adjunctive repair techniques were used in $4 \%$ to $35 \%$ of cases. The ability of the classification to predict the surgical technique 
TABLE 3. Techniques used for aortic valve repair as predicted by the aortic insufficiency classification

\begin{tabular}{|c|c|c|c|c|}
\hline \multirow[b]{2}{*}{ Class } & \multicolumn{2}{|l|}{ Predicted technique } & \multicolumn{2}{|l|}{ Adjunct techniques } \\
\hline & Description & Actual use & Description & Frequency \\
\hline \multicolumn{5}{|l|}{ One lesion } \\
\hline $\mathrm{Ia}(\mathrm{n}=22)$ & STJ remodeling, SC annuloplasty & $100 \% ; 82 \%$ & Leaflet shaving, quadricuspid repair & $4 \%, 4 \%$ \\
\hline $\mathrm{Ib}(\mathrm{n}=50)$ & AV-sparing procedures & $100 \%$ & Leaflet repair: bicuspid, tricuspid & $12 \%, 10 \%$ \\
\hline Ic $(\mathrm{n}=21)$ & SC annuloplasty & $100 \%$ & Leaflet repair & $29 \%$ \\
\hline Id $(n=6)$ & Patch repair, SC annuloplasty & $100 \%, 83 \%$ & STJ plication & $17 \%$ \\
\hline $\mathrm{II}(\mathrm{n}=54)$ & Leaflet repair, SC annuloplasty & $100 \%, 91 \%$ & STJ plication & $31 \%$ \\
\hline $\mathrm{III}(\mathrm{n}=17)$ & Leaflet repair, SC annuloplasty & $100 \%, 82 \%$ & STJ plication & $35 \%$ \\
\hline \multicolumn{5}{|l|}{ Two lesions } \\
\hline Ia and II $(\mathrm{n}=14)$ & STJ remodeling, SC annuloplasty, leaflet repair & $100 \%, 93 \%, 100 \%$ & - & - \\
\hline $\mathrm{Ib}$ and $\mathrm{II}(\mathrm{n}=38)$ & $\mathrm{AV}$-sparing procedures, leaflet repair & $100 \%, 100 \%$ & Leaflet shaving & $5 \%$ \\
\hline $\mathrm{Ib}$ and III $(\mathrm{n}=10)$ & $\mathrm{AV}$-sparing procedures, leaflet repair & $100 \%, 90 \%$ & STJ plication & $10 \%$ \\
\hline II and III $(\mathrm{n}=10)$ & Leaflet repair, SC annuloplasty & $100 \%, 100 \%$ & STJ plication & $30 \%$ \\
\hline \multicolumn{5}{|l|}{ Three lesions } \\
\hline $\mathrm{Ib}, \mathrm{II}$, and III $(\mathrm{n}=11)$ & $\mathrm{AV}$-sparing procedures, leaflet repair & $100 \%, 100 \%$ & - & - \\
\hline
\end{tabular}
STJ, Sinotubular junction; $S C$, subcommissural; $A V$, aortic valve.

was evident for patients with one, two, or three concomitant lesions.

\section{Clinical Outcome}

Clinical follow-up was complete for $99 \%$ of patients, 1171 patient-years, with a median follow-up of 47 (interquartile range 29-73) months. Echocardiographic followup was complete for $95 \%$ of patients. Midterm clinical outcomes for the entire cohort are depicted in Figure 3. Overall survivals were $95 \% \pm 3 \%$ at 5 years and $87 \% \pm$ $8 \%$ at 8 years. Freedoms from cardiac death were $97 \% \pm$
$3 \%$ at 5 years and $95 \% \pm 5 \%$ at 8 years. Freedoms from aortic valve reoperation at 5 and 8 years were $92 \% \pm 4 \%$ and $91 \% \pm 5 \%$, respectively, and freedoms from aortic valve replacement at 5 and 8 years were $94 \pm 3 \%$ and $93 \pm 4 \%$, respectively. Early complications among the patients undergoing aortic valve reoperation included 1 death, 1 postoperative myocardial infarction, and 1 reexploration for postoperative bleeding. Freedoms from AI greater than $2+$ were $88 \% \pm 3 \%$ at 5 years and $79 \% \pm 11 \%$ at 8 years. During the follow-up period, 4 patients had strokes, 1 had a transient ischemic attack, and 1 had aortic valve endocarditis.
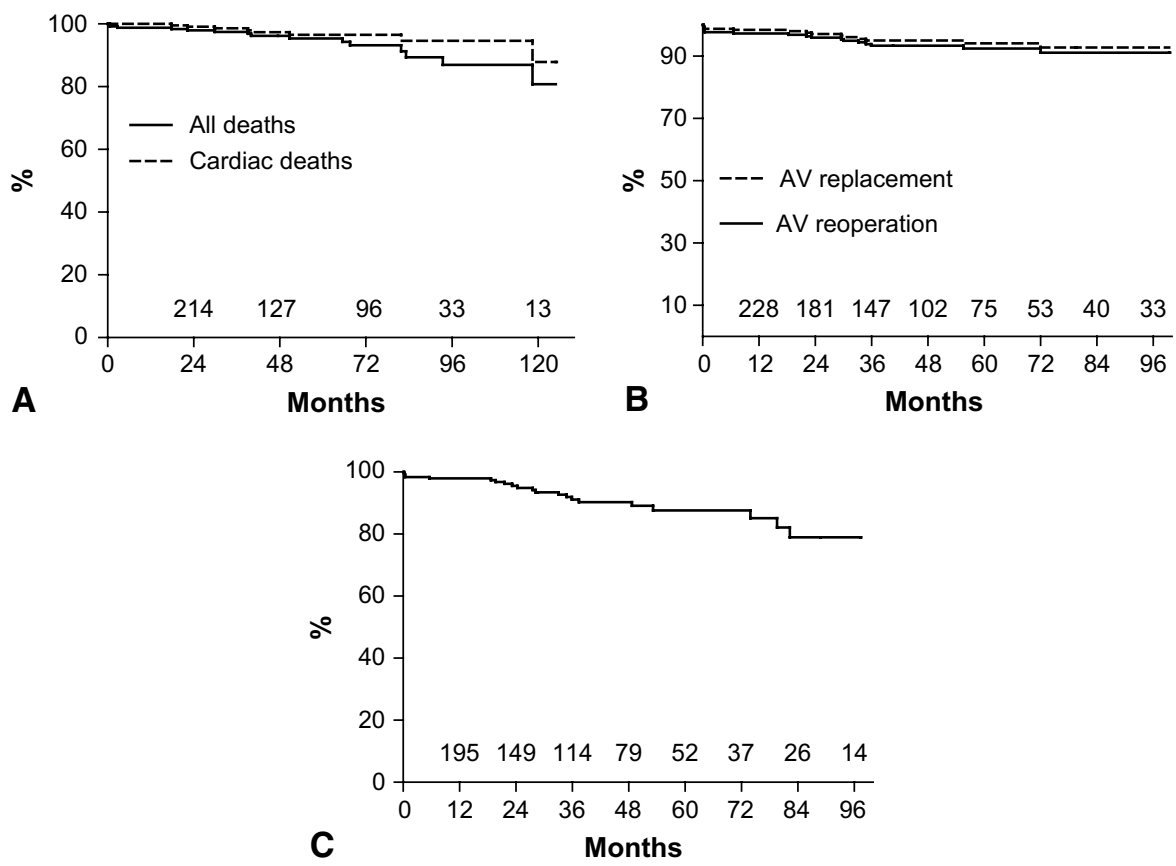

FIGURE 3. Clinical outcomes in entire cohort $(\mathrm{n}=264)$. A, Overall survival and freedom from cardiac death. B, Freedom from aortic valve $(A V)$ reoperation and replacement. C, Freedom from recurrent aortic insufficiency greater than $2+$. 
To determine whether clinical outcome could be predicted by classification of AI, we compared outcomes among the different classes of AI. At 5-year follow-up, freedoms from aortic valve reoperation were similar in types I and II and slightly reduced in type III (type I or II $94 \% \pm 4 \%$, type III $88 \% \pm 9 \%, P=.08$ ). Freedom from recurrent AI $(>2+)$, however, was significantly lower for type III AI (restrictive cusp motion) than for type I or II (hazard ratio $2.6,95 \%$ confidence interval $1.1-11.6, P=.03)$. Cusp anatomy (ie, number of aortic valve cusps) did not have an impact on midterm clinical outcome ( $P=.7$; Figure 4$)$.

\section{DISCUSSION}

Aortic valve repair techniques are infrequently used for the correction of AI. This is partly because of the inability to assess the mechanism of insufficiency systematically and apply appropriate and reproducible techniques to repair the valve. By incorporating important lessons from the Carpentier classification of mitral insufficiency, we have developed a repair-oriented classification of AI. During the past decade, we applied this systematic approach to aortic valve repair in 264 consecutive patients presenting with AI greater than $2+$. We found that early and midterm outcomes of aortic valve repair in this patient population were acceptable, with 95\% freedom from cardiac mortality, 91\% freedom from aortic valve reoperation at 8 years, and $88 \%$ freedom from recurrent AI at 5 years. Furthermore, we have demonstrated that this classification is a useful tool to describe the mech- anism of $\mathrm{AI}$ and can predict the repair techniques required in most cases. Finally, this classification also predicts clinical outcome, with type III AI caused by cusp restriction being a risk factor for recurrent $\mathrm{AI}$ at midterm follow-up. In summary, this repair-oriented functional classification of AI can help to increase the application of valve repair techniques for AI.

Classification of disease mechanisms can aid in the treatment of disease. In the case of AI, our classification system has successfully provided a common language for use among cardiologists, anesthesiologists, and surgeons in the description of AI and its mechanisms. We have previously demonstrated that there is excellent correlation between echocardiographic and surgical assessments of the aortic valve with respect to disease classification. ${ }^{8}$ Furthermore, this classification can be used to guide the repair techniques, thus standardizing the approach to the surgical repair of AI. In our cohort, we found that the vast majority of patients with either single or multiple lesions underwent repair with the techniques predicted by our disease classification. Adjunctive techniques not predicted by the classification, however, were used for as many as $35 \%$ of patients on the basis of intraoperative findings. The most common adjunctive techniques were the addition of leaflet repair in type Ic disease and the addition of STJ plication in type II and type III disease. The addition of leaflet repair in primary functional aortic annular disease (eg, type Ic) likely reflects the intraoperative discovery of previously missed leaflet pathology or induced leaflet prolapse after reduction of
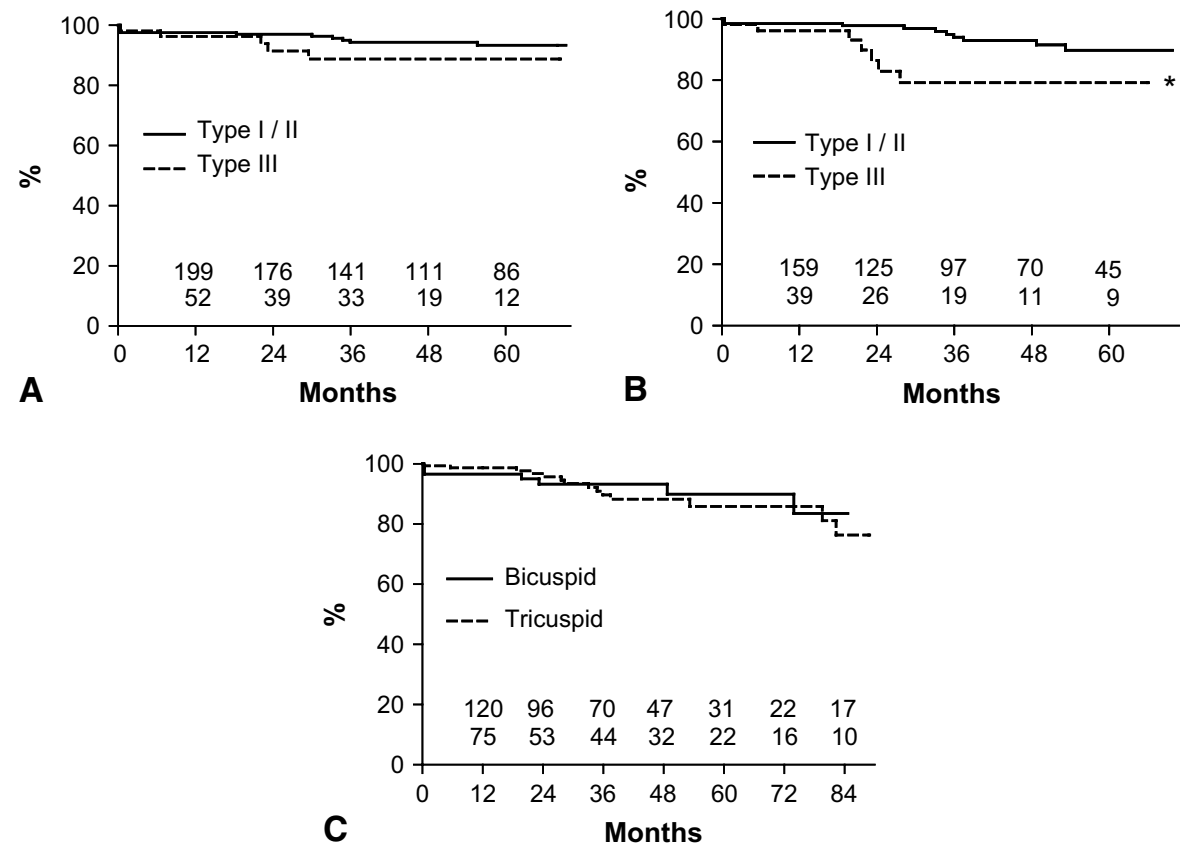

FIGURE 4. Comparative clinical outcomes of type I or II versus type III aortic insufficiency with respect to freedom from aortic valve reoperation (A, $P=$ .08 ) and recurrence of aortic insufficiency greater than $2+(\mathrm{B})$. Asterisk indicates $P=.03$. C, Comparison of aortic insufficiency recurrence in bicuspid versus tricuspid aortic valve repair $(P=.7)$. 
functional aortic annulus. STJ plication, added in about $30 \%$ of type II and III disease cases, is consistent with the notion of the two separate components of the functional aortic annulus, the VAJ and the STJ, that need to be stabilized during aortic valve repair. Overall, these findings reinforce the idea that although the key aspects of aortic valve repair can be systematically predicted by using this classification system, important roles still remain for intraoperative valve assessment and subjective decision making by the operating surgeon.

An important feature of a disease classification is the prognostic information it provides to optimize patient selection and guide treatment. In our experience, type III AI, caused by cusp restriction, was predictive of midterm AI recurrence. Although reoperation rates were not significantly different, this information is an important factor to incorporate into the decision making regarding repair versus replacement of the aortic valve. Interestingly, cusp anatomy (ie, number of cusps) had no impact on midterm clinical or echocardiographic outcome. Classification of AI also provides the ability to compare midterm and long-term clinical outcomes of different repair techniques in a comparable group of patients.

Other classification systems have been proposed for aortic valve disease. Haydar and colleagues ${ }^{9}$ presented a classification system that they applied to 44 patients with AI. Their cohort consisted primarily of young patients (mean age 33 years), with most having congenital aortic valve disease $(68 \%)$. Although they classified AI into three types (caused by annular dilatation, redundant leaflet tissue, and deficient leaflet tissue), they did not specifically classify patients with aortic root and ascending aortic pathology, which is a common cause of AI in adults, or those with cusp perforation or rupture of fenestrations. Lansac and associates ${ }^{10}$ recently reported a detailed classification system for AI that was based on postmortem assessment. Their proposed system provides limited information regarding reparability, however, because valve-sparing surgery was performed in fewer than $10 \%$ of their cases, and the degree of $\mathrm{AI}$ in that population was not reported. Furthermore, no specific correlations between echocardiographic and pathologic findings were reported. Finally, Sievers and colleagues ${ }^{11}$ have described a detailed anatomic classification of bicuspid aortic valves according to pathologic examination; this provides useful anatomic information but is not specifically geared toward repair techniques. In contrast, our classification both successfully encompasses all anatomic subsets of AI and provides insight into mechanisms and surgical treatment.

Aortic valve repair has numerous potential advantages relative to prosthetic valve replacement. First, it preserves the dynamic native aortic valve annulus and native valve tissue, which may have hemodynamic benefits relative to a rigid prosthetic valve stent. Second, avoidance of a mechanical prosthesis, which would often be used in this young population, reduces the risk of thromboembolic and antico- agulation-related complications. Finally, valve repair has been proposed to carry a low incidence of valve endocarditis. ${ }^{12}$ In 1171 years of patient follow-up, we saw only 1 case of endocarditis, which corresponds to a linearized rate of $0.08 \%$ per patient-year. Furthermore, only 5 patients in our cohort had events that could be attributed to thromboembolism, yielding a linearized rate of $0.43 \%$ per patient-year, which is approximately half the rate reported for prosthetic aortic valves. ${ }^{13,14}$ The risk of reoperation for disease recurrence is perhaps the most important factor when comparing repair with bioprosthetic valve replacement. In our experience, the risk of aortic valve reoperation was acceptable for this young cohort of patients, with a freedom from reoperation of $91 \%$ at 8 years overall. For patients with type I or II disease, the freedom from reoperation was $94 \%$ at 8 years. Similar results have been reported by others in contemporary literature.$^{12,15}$ It is important to note in this context that rates of modern bioprosthetic structural valve deterioration are significantly higher among younger patients $(3.7 \% /$ year in patients younger than 50 years and about $2 \% / y e a r$ in patients between 50 and 60 years old). ${ }^{16}$ It is likely that increasing experience with valve repair techniques, as well as optimal patient selection, will help to improve the durability of aortic valve repair. Finally, an aortic valve reoperation after repair is theoretically simpler from a technical perspective than one after placement of a stented or stentless prosthesis. Ultimately, the decision to propose aortic valve repair versus replacement needs to incorporate numerous patient- and disease-specific factors, patient preferences, and the surgeon's experience. Longer-term follow-up data will help to guide this decision making, particularly for younger patients with aortic valve disease.

\section{CONCLUSIONS}

Aortic valve repair can be performed safely and with acceptable early and midterm outcomes for patients with AI. Our proposed classification system encompasses all types of $\mathrm{AI}$, provides a common language for communication across different disciplines, guides the repair techniques used, and can help to predict midterm outcome. Type III AI, caused by cusp restriction, is a risk factor for recurrent AI. This systematic, repair-oriented functional classification can help to increase the use of repair techniques for AI.

\section{References}

1. David TE, Feindel CM. An aortic valve-sparing operation for patients with aortic incompetence and aneurysm of the ascending aorta. J Thorac Cardiovasc Surg. 1992;103:617-22.

2. Yacoub MH, Gehle P, Chandrasekaran V, Birks EJ, Child A, Radley-Smith R. Late results of a valve-preserving operation in patients with aneurysms of the ascending aorta and root. J Thorac Cardiovasc Surg. 1998;115:1080-90.

3. Burkhart HM, Zehr KJ, Schaff HV, Daly RC, Dearani JA, Orszulak TA. Valvepreserving aortic root reconstruction: a comparison of techniques. J Heart Valve Dis. 2003;12:62-7.

4. Jeanmart H, de Kerchove L, Glineur D, Goffinet JM, Rougui I, Van Dyck M, et al. Aortic valve repair: the functional approach to leaflet prolapse and valve-sparing surgery. Ann Thorac Surg. 2007;83:S746-51; discussion S85-90. 
5. El Khoury G, Vanoverschelde JL, Glineur D, Poncelet A, Verhelst R, Astarci P, et al. Repair of aortic valve prolapse: experience with 44 patients. Eur J Cardiothorac Surg. 2004;26:628-33.

6. Carpentier A. Cardiac valve surgery-the "French correction'. J Thorac Cardiovasc Surg. 1983;86:323-37.

7. El Khoury G, Glineur D, Rubay J, Verhelst R, d'Acoz Y, Poncelet A, et al. Functional classification of aortic root/valve abnormalities and their correlation with etiologies and surgical procedures. Curr Opin Cardiol. 2005;20:115-21.

8. de Waroux JB, Pouleur AC, Goffinet C, Vancraeynest D, Van Dyck M, Robert A, et al. Functional anatomy of aortic regurgitation: accuracy, prediction of surgical repairability, and outcome implications of transesophageal echocardiography. Circulation. 2007;116(11 Suppl):I264-9.

9. Haydar HS, He GW, Hovaguimian H, McIrvin DM, King DH, Starr A. Valve repair for aortic insufficiency: surgical classification and techniques. Eur J Cardiothorac Surg. 1997;11:258-65.

10. Lansac E, Di Centa I, Raoux F, Attar NA, Acar C, Joudinaud T, et al. A lesional classification to standardize surgical management of aortic insufficiency towards valve repair. Eur J Cardiothorac Surg. 2008;33:872-80.

11. Sievers HH, Schmidtke C. A classification system for the bicuspid aortic valve from 304 surgical specimens. J Thorac Cardiovasc Surg. 2007;133:1226-33.

12. Aicher D, Langer F, Adam O, Tscholl D, Lausberg H, Schafers HJ. Cusp repair in aortic valve reconstruction: does the technique affect stability? J Thorac Cardiovasc Surg. 2007;134:1533-9.

13. Peterseim DS, Cen YY, Cheruvu S, Landolfo K, Bashore TM, Lowe JE, et al. Long-term outcome after biologic versus mechanical aortic valve replacement in 841 patients. J Thorac Cardiovasc Surg. 1999;117:890-7.

14. Ruel M, Masters RG, Rubens FD, Bedard PJ, Pipe AL, Goldstein WG, et al. Late incidence and determinants of stroke after aortic and mitral valve replacement. Ann Thorac Surg. 2004;78:77-84.

15. David TE, Feindel CM, Webb GD, Colman JM, Armstrong S, Maganti M. Aortic valve preservation in patients with aortic root aneurysm: results of the reimplantation technique. Ann Thorac Surg. 2007;83:S732-5; discussion S85-90.

16. Jamieson WR, Burr LH, Miyagishima RT, Germann E, Macnab JS, Stanford E, et al. Carpentier-Edwards supra-annular aortic porcine bioprosthesis: clinical performance over 20 years. J Thorac Cardiovasc Surg. 2005;130:994-1000.

\section{Discussion}

Dr Hans-H. Sievers (Lübeck, Germany). First, I must disclose my financial relationship to the companies of Sorin (Italy) and Aesculap (Germany).

Dr El Khoury, I congratulate you on an outstanding study and especially for your great efforts to promote aortic valve reconstruction, which is an appealing operation improving in results and techniques. Nevertheless, some results are suboptimal, and a lot of questions are open. You could nicely demonstrate that a seemingly simple repair-oriented classification system of AI supports the understanding of functional anatomy for standardization of reconstructive techniques. Also, your classification system, which combines surgery and anatomy, is useful to direct the choice of the operative method in general. More details, however, are important for decision making and the success of the operation. Briefly, precise definitions are desirable. Thus my first question is as follows. Your classification type I refers to dilatation of different levels of the root. What exactly do you mean by dilatation? You measured the diameters by echocardiography and intraoperatively, but what are your threshold values for diameters to call it dilatation, which is important for decision making?

Dr El Khoury. Thank you, Dr Sievers. The concept of functional aortic annular dilatation is helpful in determining the surgical techniques for this strategy. When we have functional aortic annular dilatation, we have aortic regurgitation; conversely, when we have aortic regurgitation, I believe we have some kind of functional aortic annular dilatation. Saying that, type Ia and type Ib are the classic aneurysmal dilatation of the aneurysmal descending aorta and the root, and we use the standard measurement as the indication for surgery. If we look at types Ic, Id, and even II, I support that there is functional aortic annular dilatation. We have to look at it as a mismatch between the quantity of leaflet present and the aortic orifice. So the idea is that when we have this mismatch between the quantity of tissue and the orifice, we have two ways to restore the match: either extend the leaflet with cusp extension, or reduce the functional aortic annulus. So for types Ia and Ib, it is the classic definition of aneurysm, but for types Ic, Id, and II, it is really the idea that when we have regurgitation, we have some kind of mismatch, and the idea of valve repair is to restore the match between the leaflet and the aortic orifice.

Dr Sievers. Second, when you assess the aortic valve for the mechanisms of AI, especially in type II insufficiency, do you use special tricks or instruments or sutures to imitate the shape of the root at diastolic pressure to decide which of the various techniques to apply?

Dr El Khoury. After the standard transverse aortotomy, I use a systematic approach. After the transverse aortotomy $1 \mathrm{~cm}$ above the STJ, I put three-sutures at the commissures, and I put traction on those three sutures. First, I inspect, and sometimes in the first inspection I can see which leaflet is prolapsing or if the three leaflets are at the same level. So, the first step is inspection. If I am not happy with the inspection, I have to know the appropriate level of the leaflet. If one looks at the normal aortic valve in an echocardiogram, if this is the STJ and this is the leaflet, if one looks at the echocardiogram, the level of the free margin is really at the mid height of the commissure or mid height of the sinus of Valsalva. So when I open the aorta, I put traction on the three commissures, and with the forceps at the middle of the Arantius node, I can push down the leaflet and see at which level each leaflet goes down and whether the three leaflets are at the same level. This is one way I use.

The second way I use is to put a 7-0 or 8-0 suture at the middle of the cusps and pull up. Usually the free margins are running parallel when the leaflets are normal, but if one looks on the prolapsing one, the free margin is not parallel. So the nonparallel free margin is the prolapsing one. I don't use any instruments.

Dr Sievers. The last question is as follows. I had to reoperate on some of our 430 reconstructed aortic valves for subcommissural annuloplasty failure, but only in patients with a bicuspid valve, not a tricuspid. So at least in my hands, there seems to be a difference concerning subcommissural annuloplasty and valve etiology. Do you think it makes sense to consider valve etiology in your repair-oriented classification system?

Dr El Khoury. We were taught by Professor Carpentier that cardiac surgeons usually don't care about etiology. We have to restore the function of the valve.

The bicuspid aortic valve in our classification is type II or type I. So it can be type Ia, Ib, or whatever. This is mainly Ia and Ib, and I think that it is Ic in the pediatric population. I am aware of your classification for bicuspid aortic valve. And we had a discussion in New York at the Aortic Symposium about the indication for surgery and the size of the aortic root and bicuspid, tricuspid, whatever. But I think we have missed one thing, that is the perioperative assessment and examination of the quality of the aortic wall. Mainly in the 
bicuspid aortic valve, even if the aortic root is normal, we can find that the leaflet at the insertion to the aortic wall is too transparent, too thin, and we can see even the muscle. So in those cases, I think we have to be more aggressive and not do subcommissural annuloplasty but go to the valve-sparing operation, the David operation or whatever. I think that diameter is not enough for the indication to replace the ascending aorta with a bicuspid aortic valve. We also have to look the quality of the tissue.

Dr Christopher M. Feindel (Toronto, Ontario, Canada). First, I congratulate you for trying to put some methodology and organization to what still seems to be an eyeballing technique. I think this is a great help.

I do have a question about type III and your efforts to decalcify valves. I must say, years ago I learned painfully that this was a lousy operation, and we ended up reoperating on every one of those patients.

Dr El Khoury. With regard to type III, we have two kinds. We have type III associated with bicuspid aortic valve when we have the raphe, so we can resect the raphe. This is one thing. Type III with a tricuspid aortic valve, however, in our experience is differ- ent. I repaired everything some years ago, but now I really limit in type III when I see, for instance, coronary artery surgery and moderate aortic regurgitation and moderate stenosis. The valve really doesn't move very well, so in those patients we replace or repair the valve. In many, many of those patients, really almost all the patients, we go and do some shaving, clean the valve, and add subcommissural annuloplasty.

Dr Feindel. And do you not think that those patients come back faster for reoperation but now with, in many cases, working bypass grafts?

Dr El Khoury. I am not sure that we are accelerating the process. You can do nothing, and maybe the patient will come back for repair the valve. I am not sure that we are really accelerating the process of calcification by only shaving.

Dr Feindel. Our experience has been different. We have found that we ended up reoperating on those patients 3, 4, 5 years down the road, whereas with a bioprosthetic valve, the patient has at least 10,12 , or 15 years.

Dr El Khoury. It would be useful to conduct a randomized study. 